\title{
Alternative Medicine: New Ways to Treat Diseases and Therapies
}

\author{
T. R. MAMIDI*, MATCHA YAMINI, V. S. P. CHERUKURI AND S. R. YARRAGUNTLA \\ Department of Pharmaceutics, Vignan Institute of Pharmaceutical Technology, Duvvada, Visakhapatnam
}

\section{Rao et al.: New Ways to Treat Diseases and Therapies}

\begin{abstract}
Alternative medicine is the most widely used remedy systems, in the treatment of various diseases. Alternative medicine is used widely because there are a large health care alternatives to be more congruent within the own values and beliefs towards health. Alternative medicine is more compatible with patients, offers more personal autonomy and control over the health care decisions. It is accepted worldwide because of its compatibility and acceptability in increasing the beliefs regarding the nature and meaning of health and illness. This review deals with various alternative therapies used such as Ayurveda, homeopathy, acupuncture, naturopathy, yoga, herbal medicine, massage therapy; effectiveness of the alternative medicine; assessment of the effectiveness of alternative medicine; sources of information about the alternative medicine; alternative medicine therapies in treatment of various diseases; perceived benefits of alternative medicine and thereby concluding with the increased level of acceptance of alternative medicine, its widespread use in various diseases and their treatment with various alternative therapies.
\end{abstract}

Key words: Alternative medicine, Ayurveda, homeopathy, acupuncture, naturopathy, yoga, herbal medicine, massage therapy, diseases, treatment

Alternative medicine is a type of any health care remedy or a system which is not accepted generally in the modern biomedicine or therapies that are usually offered in place of or as a substitute for the conventional therapies ${ }^{[1]}$. The main stream of medical practice usually includes the Allopathic medicine. The Various alternative therapies used are: homeopathy, acupuncture, naturopathy, herbal medicine, diet fads, folk medicine, faith healing, Ayurveda medicines, chiropractic, yoga, massage therapy and music therapy. Courses in the alternative medicine are also known as complementary medicine; private medical insurance agencies increasingly include the alternative medicine options among their coverage. In many countries alternative medicines and the therapies are usually regulated with almost no requirements to prove the efficacy or long term safety. Alternative medicine industry is booming ${ }^{[2]}$ and many companies selling alterative medicines appear to have escalating share prices on the world stock markets. The term complementary medicine and the alternative medicine refer to a set of health care practices that are not a part of the country's own traditional or the conventional medicine and are not fully integrated into the dominant health care system. In some countries they are used interchangeably with

*Address for correspondence E-mail: thrinadh_81@rediffmail.com

January-February 2021 traditional medicine in some countries. The public is becoming aware of the alternative medical therapies through all forms of the media and large proportions of the public self-prescribe alternative medicines and also by visiting the alternative practitioners in Europe, the United States and Australia ${ }^{[3,4]}$. Many patients consult their doctor or the alternative health care providers such as the naturopaths, herbalists, chiropractors, homeopaths and the practitioners of Chinese or the Ayurveda medicine for the alternative medicine therapy. Pediatricians are becoming usually more open to alternative medicine. Forty two percent of Canadians use Alternative medicine therapies ${ }^{[5]}$. Alternative medicine is used not so much because of their dissatisfaction with the conventional medicine but largely because that they find these health care alternatives to be more congruent with their own values and beliefs towards health and life. The therapies have experienced the highest increase in use including the

This is an open access article distributed under the terms of the Creative Commons Attribution-NonCommercial-ShareAlike 3.0 License, which allows others to remix, tweak, and build upon the work non-commercially, as long as the author is credited and the new creations are licensed under the identical terms

Accepted 22 February 2021

Revised 30 December 2020

Received 10 September 2020 Indian J Pharm Sci 2021;83(1):1-12 
homeopathy, energy healing, massage, mega vitamins, self-help groups, folk remedies and herbal medicine.

\section{ALTERNATIVE MEDICINE AND ITS EFFECTIVENESS}

Alternative medicine has a widespread use by over 40 percent of the total U.S population which has been a considerable challenge to the conventional health care practitioner which has shown a necessity to appreciate the different healing approaches of conventional medicines and many complementary and alternative therapies and the understanding of how these differences affect the treatment approaches and the measurement outcomes. In addition one must understand how the conventional research methodologies and the evidence reporting limit or conflict with the ability to accurately assess the effectiveness of alternative therapies and where to find reliable information about such therapies. Effectiveness of care usually implies the positive outcome of the treatment which is the result of combination of the factors like efficacy of an intervention, the approach to the patient, response of the patient and setting in which care is given. Effectiveness also reflects the aspects of placebo or the expectancy effect. Due to the costs, safety issues and the dominance of the pharmaceutical interventions, the demonstrating efficacy relied heavily on a single tool for the measurement of a biological therapeutic effectRandomizes controlled clinical trial (RCT). The RCT approach to determining of all the clinical merits of the alternative invention are usually important, given the highly individualized and the multifaceted treatment protocols typically employed. The RCT research technique usually tests a well specified and standardized treatment, delivered uniformly in a standard setting to specified subjects, designed for those who ideally have only the target condition being treated and also includes agreements with subjects to comply and adhere to the protocol usually for a reward. Due to this design the efficacy studies specifically exclude the multiple, real world clinical factors which include the approach to the patient, treatment setting, life style issues and the adherence to medication protocol. The model of the single intervention clinical trials are more useful for the pharmaceutical treatments than for assessing the outcomes of the most alternative medicine therapies where treatment protocols are tailored to individuals rather than a standard group of subjects. Assessment of the alternative therapy effectiveness may require a different approach for the gathering and interpreting evidence. In the clinical world of the alternative practices the treatment is not typically single drug or intervention, but is a complex therapeutic modality that also may involve physical and emotional interaction with the therapist.

\section{ASSESSING THE EFFECTIVENESS}

Assessment depends on the clinician's healing paradigm, such as the biomedical, homeopathic, or naturopathic. The effect of philosophy of healing such as self-healing, systems approach, balance and holism; the effect of clinician-patient relationship; the set of therapeutic methods used to enhance the healing process all the three effects play an important role in the assessment of alternative medicines and complementary medicines treatments' effectiveness. The healing philosophy on the evaluation of the outcomes is usually offered by the homeopathy. In some cases the clinicians strongly reject the scientific conclusions of the effectiveness of homeopathy therapy because the non-material nature of homeopathic remedies is outside the conventional scientific paradigm and is dismissed as placebo.

\section{EFFECTIVENESS WITH THE POWER OF BELIEFS}

Patient's beliefs regarding the treatments and medicines usually play an important role in any healing setting. The patients centered approach fits the model described by Benson 1997, for obtaining the nonspecific placebo effect. This effect is usually produce by the health professional's style and clinical setting rather than a placebo or chemical effect of the intervention such as the manual therapy or the acupuncture needling. The success of the alternative therapy practitioners is achieved by maximizing the non-specific placebo response for many of the complementary and the alternative medicines practitioners, it is a goal of the therapy to engage the patient including emotional and physical expectations in the healing process which may be more difficult to control for the placebo effect in the study design. Other beliefs also influence the success of a treatment or therapy.

\section{EXAMPLES FOR THE ASSESMENT OF EFFCTIVENESS OF ALTERNATIVE THERAPIES}

An example of an analysis of the weight of evidence in the alternative and the complementary medicines from the article Rheumatology, 'Herbal Medicines for the treatment of Osteoarthritis: A systematic review' published in the journal. This article also includes the treatment of osteoarthritis with Nonsteroidal anti- 
inflammatory drugs (NSAID's) by the authors of (from Exeter University, UK and the University of Maryland School of Medicine, US) causes about 2000 deaths a year. The search for the literature of RCT's of herbal remedies for the osteoarthritis was done and found those 12 trials and 2 systemic reviews of which some of these were unreplicated studies. A set of Eleven remedies were studied: Articulin-F (an ayurvedic herbal mineralformulation); avocado/soybean unsaponifiables (ASU); capsaicin (derived from hot chili peppers, applied topically); devil's claw (African plant, active agent: iridoid glycoside); eazmov (Ayurvedic herbal mixture); ginger; gitadyl (combination of feverfew, aspen and milfoil); phytodolor (three herb combination); reumalex (white willow bark, guaiacum, black cohosh, sarsaparilla and poplar bark); stinging nettle and willow bark. After reviewing the weight of evidence the following recommendations were made: moderately strong ( $3+$ favorable trials) evidence for the phytodolor, capsaicin. Weak evidence (1 trial with favorable outcome) for reumalex willow bark, stinging nettle, articulin-F. No evidence (no positive trials for eazomov, gitadyl, ginger extract). Finally, stated that there are some of the promising alternative and complementary options for the clinical care.

There is evidence supporting the efficacy and effectiveness of a number of alternative therapies and complementary therapies for different health conditions. The following table summarizes the efficacy and the effectiveness of the selected therapies for common clinical problems for which some evidence has accumulated (a positive direction of evidence indicates that the treatment provides benefit, a negative direction indicates no benefit for the clinical problem). Summary of the alternative and the complementary medicine effectiveness for selected medical conditions (Table 1).

\section{PRINCIPLES OF HEALING OF VARIOUS ALTERNATIVE THERAPIES}

The complementary and the alternative medicines therapeutic systems emphasize some or all of the following principle to a greater degree than the conventional medicines. Effective integration of these therapies and also the conventional care must rest on the acknowledgment, appreciation and application of these principles in patient-centered context. They provide a framework for understanding the complementary and alternative therapy approaches to healing that contrast with the biomedical model of care: Emphasize effective communication between the patient and healer, which builds the trust and hence promotes integration; Promote the body's self-healing abilities (important principle); Emphasize the self-care and empowerment of the patient in the healing process; Recognize the mind, body and spirit as interactive and inseparable; Address underlying causes of illness-including the environmental emotional and spiritual factors rather than just the clinical manifestations; Prevent ill health by remaining in balance and harmony with the psychosocial and physical environment; Enhance wellness with the optimal diet, exercise and reduced stress life style; Individualize the treatment to particular patient, rather than focusing on the disease condition; Emphasize the use of natural non-pharmaceutical substances or non-surgical techniques in the care of patient; Appreciate the electromagnetic and energetic nature of the human organism and the importance of vitality in healing; Acknowledge the healing journey and that the return to wholeness can be a gentle and gradual developmental process.

\section{SOURCES OF INFORMATION ABOUT ALTERNATIVE MEDICINE ON INTERNET}

The internet resources usually provide the information and satisfy the needs of both health care workers and physicians. The following list is useful starting point and accessing these websites leads to different alternative medicines websites.

\section{Metadirectories:}

The metadirectories that offer hyperlinked listings of the alternative medicine websites are-The Alternative medicine Home Page www.pitt.edu/ cbw/altm. $\mathrm{html}$ provides the information about the alternative medicines with access to the databases, internet resources, mailing lists and government resources. The site Alternative Medicine Health Care Information Resources-McMaster University $<$ www-hsl.mcmaster. ca/tomflem/altmed.html $>$ offers access to the general resources on alternative medicines on the World Wide Web. Links to the pages are inclusive or encyclopedic in nature. Each link usually offers access to multiple alternative medicines topics. The links to inclusive resources are followed by a list of very specific resources on single topics. This is an excellent site to access and also has many appropriate hyperlinks. The site National Center for Complementary and Alternative Medicine (NCCAM) National Institutes of Health (NIH) is sponsored by the office of Alternative Medicine established in 1992 as a part of the National Institutes of Health (Table 1). NCCAM was established 


\begin{tabular}{lccc}
\hline Problem & Therapies & Evidence strength & Evidence direction \\
\hline Alzheimer's disease & Ginkgo & Low to moderate & Uncertain \\
Anxiety & Kava, relaxation, aromatherapy & Moderate & Positive \\
Asthma & Hypnosis, homeopathy, yoga & Low to moderate & Positive \\
Eczema & Prime rose oil, Chinese herbs & Moderate & Uncertain \\
Back pain & Acupuncture, massage, manual therapy & Moderate & Positive \\
Chronic Fatigue & Exercise, herbals, homeopathy & Low to moderate & Positive \\
Constipation & Biofeedback, massage, herbals & Low to high & Positive \\
Depression & St. john's worth, relaxation, music & Low to high & Positive \\
Fibromyalgia & Exercise, homeopathy, biofeedback & Low to moderate & Positive \\
Hypercholesterolemia & Diet, garlic, gaur gum, red yeast rice & High & Positive \\
Insomnia & Kava, valerian, relaxation, melatonin & Moderate & Positive \\
Migraine & Acupuncture, bio feedback, feverfew & Moderate & Positive \\
Osteoarthritis & Acupuncture, devil's dow, glucosamine & Moderate & Positive \\
Palliative care & Acupuncture, healing touch & Low & Positive \\
Prostatic Hyperplasia & Palmetto, African plum & Positive & \\
\hline
\end{tabular}

to 'facilitate the evaluation of alternative medical treatment modalitie's ${ }^{[6]}$ to determine the effectiveness. NCCAM conducts the applied research and training and it disseminates information on the alternative medicine and the complementary medicine practitioners and the public and also brings the researchers together to prepare grant applications. With the united states food and drug administration the NCCAM holds regular meetings to enlist its cooperation in re-evaluating current rules and regulations governing research about devices and their use, acupuncture needles, herbs and homeopathic remedies and corresponds with many alternative medical organizations and provide information about research support and development. The site Alternative Health News Online www.altmedicine.com/ corresponds to the statement 'be aware that alternative health and healing covers everything from pure hogwash to promising and proven therapies. This helps the consumer evaluate the alternative medical approaches.

\section{Databases:}

Databases allow the users to search for articles on specific topics regarding alternative medicines. Alt-Health Watch www.softlineweb.com/althealth includes the full text database of peer-reviewed journals, periodicals, academic and professional publications, magazines, consumer newsletters and newspapers, research reports and the association newsletters focused on alternative, complementary and integrated approaches to health care. Alt-Health Watch provides the information regarding the professional and consumer levels across the full spectrum of 200 therapies, modalities and the various perspectives addressed by integrated medicine. The International Bibliographic Information on Dietary
Supplement (IBIDS) <odp.od.nih.gov/ods/databases/ ibids.html $>$ is the database of published, International, scientific literature on dietary supplements, including the vitamins, minerals and botanicals. This database contains 300000 scientific citations and abstracts. It provides a journal list of more than 1500 publications with links to websites where the users may order the full journal articles. Southwest school of Botanical Medicine-Plant Abstracts includes the European and Asian plants and provides an alphabetical listing of plants that have received some investigations. The abstracts from the foreign medical and pharmacological journals include both human and animal research.

\section{VARIOUS ALTERNATIVE THERAPIES AND THEIR TREATMENT ON VARIOUS DISEASES}

\section{Ayurveda:}

Ayurveda (ayush=life, vid=wisdom-Sanskrit) is the ancient medical systems within ancient Vedic tradition offering the wealth of knowledge for meaning full life and health ${ }^{[7]}$. Ayurveda, part of atharvaveda, the last one of the four Vedas is timeless and unbounded it has relevance even to the present day. The philosophy of Ayurveda believes that everything in this universe is composed of five elements, the panchabhootas. They, in turn constitute the three doshas or bio-energetic forces that govern our health and determine our constitution. Mental and the spiritual health are determined by the trigunas or psychic forces. The modern risk factor hypothesis pales into insignificance in the view of most elaborate knowledge in Ayurveda.

The recent additions to the modern medical list like 
hostility from the basis of Ayurveda risk factors. Viz. Krodha, sokha, bhaya, aayasa, virudhaannabhojana, Thaponnalaan, lavanakshara... rekta pitta prakopayeth." (Anger, depression and sorrow, extreme fear, exhaustion, wrong foods, sedentary living, too much salt and spices lead to most of the major killer diseases).

The solution for this is Nithyahitha, mithaaaharasevi, sameekshkaari, dhatasamahasathyapara Kshamavaana apthopaseviaarogyam."(Have food in moderation and also that which pleases the mind. Do not cheat tell lies. Work very hard, forgive others even if they hurt you. Treat all as your own kith and kin. Health would be yours always $)^{[8]}$.

Vedic wisdom is the right approach for holistic assessment of health and disease. The Vedic wisdom aims not only to heal the sick, but also to preserve life by preventing illness-the most complete system of health care known. It follows non-linear mathematics. Ayurvedic treatment depends on restoring the distributed balance of the three dosha-vata, pitta and kapha. Ti considers the whole human being not only the phenotype. It includes the genotype as also the mind in classifying patients. Based on the constitutional types treatment differs from individual to individual even for the same disease. Ayurveda astrology usually plays an important role in disease and its cure but the science of astrology is good but not the astrologers' predictions, most predictions including those in the scientific predictions are just "predicting the unpredictable"[?]

The long term prospective studies from the basis of therapy in Ayurveda, includes the treatment tailored to individual needs and is broadly grouped into the following:

Detoxification-panchakarmas, diet, yoga, herbal medications, meditation and prayer (no particular god or book prescribed), Ayurveda surgery.

Sushruta described the various advances in Ayurveda surgery which could be gauged from the intricate rhinoplasty, thousands of years ago. Stone removal and trephining from various sites, hysterectomy, abdominal child birth, episiotomy, fracture setting, tumor surgery, minor surgical procedures has been described. They had 127 instrument and suture materials. Anesthesia, sanmohini, was the much more advanced and aseptic methods were the most modern. The lancet used for small pox vaccinations resembles the one used in the modern medicine. Jenner's vaccination has gained the authentication from the ancient ayurvedic vaccination's proven track record. Dr. T. Z. Holwell, presented the various details of ancient Indian vaccination methods to the London Royal College of Physicians on $1747^{[10]}$. Various detailed texts existed for the physiology, symptomatology, surgery, drug therapy and diet. The descriptions of the angina pain and its origin were explained ${ }^{[11]}$.

"Athaathohradrogaprathishedamvyakyawamyah

YathovaachaBaghavanDhanvantharim:

"AyaammyatheMaaruthajeHradayamThudyathe,

NirmaathyatheDheeryathe Cha Spotyathe,

PaatichaThrinoshadaahachoshaamsyuhuPaithikecha,

Dhoomaayam Cha Moorchaa Cha Swedhahako."

In the above chapter the symptoms of heart diseases and impending death by heart attacks is described by Bhaghvan Dhanvanthari (God of Healing). There are eight branches of Ayurveda integrated at all levels: Surgery, medicine, gynecology, pediatrics, toxicology, otorhinolaryngology, rejuvenation and the virilification therapy.

The oath of Indian physician is much more elaborate compared to that of the Hippocratic Oath. In a classic, the Greek historian described how Aryans (cultured men-Sanskrit) migrated from India to Greece via Sumeia and many other countries ${ }^{[7]}$. History shows the much data how the ancient Indian texts and pundits were taken to Greece by the army of Alexander the Great. Charaka Samhitha and the Susrutha Samhitha, were the most famous of all the texts, many others like MadhavaNidhaana are also said to be significant. Several thousands of medicinal plants mentioned in the ancient texts hold the key to future inexpensive pharmacology. Charaka, the great Ayurveda sage wrote. "That is designated as Ayurveda or the science of life wherein are laid down the good and bad of life, the happy and unhappy life and what is wholesome and what is unwholesome of life, as also the measure of life" "[12]. In order to complement modern medicine whitins all of its disadvantages, Ayurveda fits the bill perfectly with less expensive and equally effective methods good for long term care and is highly effective. Ayurveda is not just an alternative medicine but the key basis of India's health.

\section{Homeopathy:}

Homeopathy is defined as the therapeutic method using preparation of substances whose effect when administered to healthy subjects correspond to the manifestations of the disorder (symptoms, clinical 
signs and pathological states) in the individual patient. Homeopathy as an alternative and complementary medicine and a holistic medicine can solve some of the modern medical problems. This method was developed by-Samuel Hahnemann (1755-1843) a German physician in 1810 and treatment for diseases with about 3000 homeopathic remedies was addressed based on the golden principles and now it is practiced throughout the world ${ }^{[13]}$. Homeopathy is based on two main principals ${ }^{[14,15]}$. The first principle-'like cures like principle' (similiasimilibuscurentur), states that patients with particular signs and symptoms can be helped by a homeopathic remedy that produces there signs and symptoms in healthy individuals. The second principle states that homeopathic remedies retain the biological activity after repeated dilution and succession even when diluted beyond Avagadro's number. The origin of homeopathic medicines includes the following percentages of Herbal $>80 \%$, Salts $10 \%-15 \%$ and Animal about $5 \%$. The Food and Drug administration of the United States of America in 1938 recognized all the Homeopathic remedies as "medicine", not a supplement or vitamin. Homeopathic remedies were proven to be effective, safe and cost-effective. After this study Switzerland joined the countries of Germany, England, France, Denmark and Luxembourg and provided the homeopathic remedies under insurance ${ }^{[16]}$. Now a day's homeopathy is recognized as the second important medicine of world in terms of the extent of the patients covered. $40 \%$ of the French people use these homeopathic medicines for common cold and allergies. In Germany, the most commonly used treatment for Hay Fever (a seasonal sensitivity) is homeopathy ${ }^{[17]}$.

In the $200 \mathrm{y}$ history critics have pointed out that its very principles fly in the face of science, while proponents have maintained that it is narrow minded to reject an overtly helpful approach to healing only because cannot explain how it might work ${ }^{[14]}$. Various conclusions have been given for the homeopathic medicines describing them that they are more than mere placebo and superior to placebo by Linde in $1997^{[15]}$. Despite this and other caveats homeopaths worldwide have celebrated this publication the ultimate proof of their treatment. Since then, a flurry of interest in homeopathy has emerged. Since 1997 with a view to defining the clinical effectiveness of homeopathic medicines has emerged.

A scientist named Cohort revealed that homeopathy could effectively reduce patients' disease severity and improve their quality of life ${ }^{[18]}$ from his study on 3981 patients with allergic rhinitis, headache and atopic dermatitis. Homeopathy could significantly decrease the pulmonary discharge in patients with Chronic obstructive pulmonary diseases (COPD) who admitted in the Intensive care unit (ICU) and mechanically ventilated and the patients were separated and discharged from the ventilator device faster than the control $\mathrm{arm}^{[19]}$. The effect of Homeopathic remedies efficacy on animals and herbals has been proven and these evidences discourage induction in homeopathy (the use of Blatta orientalis in Indian guinea pig and rat has been led to reduction of acetyl choline, histamine, eosinophil, immunoglobulin $\mathrm{E}(\mathrm{IgE})$ and mast cell in hypersensitive lungs accompanied with bronchospasm ${ }^{[20]}$. Gamma interferon production rate by $\mathrm{T}$ cells has been increased up to $24 \%$ after the homeopathic remedies use. This also discourages the induction in homeopathy ${ }^{[21]}$.

\section{Acupuncture:}

Acupuncture means "to puncture with a needle". The term acupuncture implies two different words from Latin: acus means needle and puncture means insertion $^{[22]}$. It is the best known of alternative and complementary therapies. Acupuncture is a treatment method that originated more than $3000 \mathrm{y}$ ago in China and also practiced in most of the world ${ }^{[23]}$. Traditional Chinese medicine defines acupuncture as: the stimulation of certain points on or near the surface of the human body through any technique of point of stimulation with or without the insertion of needles, these include the use of electrical, magnetic, light and sound energy, cupping and moxibustion (the burning on or over the skin of selected herbs), to normalize the physiologic functions or to treat various conditions of the human body ${ }^{[24]}$. The practice of acupuncture consists of inserting fine, solid needles (usually 32 to 36 gauges) into selected body locations (acupuncture points). It describes there are 365 points located in systematic fashion on meridians or "channels of energy flow" that are mapped onto the surface of the body. The key principles in traditional Chinese medicine (TCM) are that both wellness and illness sult from an imbalance of yin and yang. Yin refers to the feminine aspect of life: nourishing, lower, cool, deficient, inside, receptive, protective, soft and yielding. Yang is the male counterpoint: hard, dominant, energetic, upper, hot, excessive, outside and creative. The movement between these opposite forces, named Qi, is considered to be the essential element in the healing system of TCM. Within the wide usage of acupuncture, patients self-refer to acupuncturist for a variety of indications. 
The National Institutes of Health $(\mathrm{NIH})$ convened a consensus panel to review the available literature about acupuncture $^{[25]}$, clinical efficacy and effectiveness, biological effects implications on health care system and the need for further research. Further the acupuncture research trials have been funded by the NIH/NCCAM and other agencies. Various national institutes of health consensus panel on acupuncture ${ }^{[26]}$. Skeptics maintains that acupuncture has basically a placebo effect since, acupuncture meridians and their "energy" or "chi (Qi)" as described in the TCM cannot be directly observed, dissected or measured with standard anatomic approaches or physiologic instrumentation. The acupoints are located at sites that have a high density of neurovascular structures, between or at the edges of muscle groups ${ }^{[27]}$ and these are of less painful than random needle sticks into the muscle group. A study demonstrated that needling a point on the lower leg traditionally associated with eye activated the occipital cortex of the brain as detected by functional magnetic resonance imaging ${ }^{[28]}$. The explanation of TCM system of medicine, including the effects of acupuncture, is rich with metaphor and allegory ${ }^{[29]}$. These explanations refer to different kinds of Qi, the influence and interaction of the five elements (fire, earth, metal, water and wood) yin and yang and other terminology that requires contemplation and long study of a culturally distinct system. Other organizations have also addressed the potential benefits of acupuncture. The World Health Organization has identified more than 40 medical conditions treated effectively with acupuncture. The various indications for acupuncture by world health organization ${ }^{[30]}$ : Respiratory tract acute sinusitis, acute rhinitis, common cold, acute tonsillitis; Gastrointestinal disorders spasm of the esophagus and cardia, hiccup, gastroptosis and chronic gastritis, gastric hyperacidity, chronic duodenal ulcer (pain relief), acute and chronic colitis, constipation, diarrhea, acute bacillary dysentery, paralytic ileus; Bronchopulomonary disorders-acute bronchitis, bronchial asthma; Neurological disordersheadache, migraine, trigeminal neuralgia, facial palsy, paresis After stroke, peripheral neuropathiesis, Meniere disease, Sequelae of poliomyelitis (early stage), Meniere disease, Neurogenic bladder dysfunction, nocturnal enuresis, Intercostal neuralgia; Disorders of the eye-acute conjunctivitis, central retinitis, myopia (in children) Cataract (without complications); Musculoskeletal disorders-cervicobrachial syndrome, frozen shoulder, tennis elbow, Sciatica, low back pain, osteoarthritis; Disorder of mouth-toothache, post extraction pain, gingivitis, acute and chronic Pharyngitis.

Overall in the United States, acute and chronic musculoskeletal indications for acupuncture treatments have found greatest acceptance. Acupuncture may be useful in difficult conditions such as asthenic states ("tired all the time", "low energy"), autonomic dysregulation disorders (Anxiety, sleep disturbance, bowel dysfunction) and immune dysregulation disorders (recurrent infections and inflammations) [31]. In many acupuncture practices, the most common indications are mainly for chronic pain unresponsive to standard therapy. The ancient method of acupuncture has gained significant popularity in our era, most of the patients select acupuncture as part of their therapeutic plan because if it's long history of use, safety and reports of efficacy. Physicians may also wish to expand the scope of their practice by taking additional training to administer acupuncture, even if one doesn't add this training, knowing how to refer the credible, well trained acupuncturists and for what indications is increasingly important in evolving model of integrative, combining the best of both scientific medicine and traditional systems of care.

\section{Naturopathy:}

Naturopathic medicine is a distinct method of primary health care-an art, science, philosophy and practice of diagnosis, treatment and prevention of illness ${ }^{[32]}$. It is a distinct type of primary health care medicine that usually blends age-old healing traditions with scientific advances and current research. It is guided by unique set of principles that recognize the body's innate healing capacity, emphasize the disease prevention and encourage individual responsibility to obtain optimal health. Principles of naturopathic medicine: The healing power of nature-naturopathic recognizes the body's natural healing ability and trusts that the body has the innate wisdom and intelligence to heal itself given the proper guidance and tools; Identify and treat causes-ND's attempt to identify and treat the underlying causes of illness, rather than focusing on individual presenting symptoms; First do no harmND's begin with minimal interventions and proceed to higher level interventions only as determined necessary; Doctor as teacher-ND's educate patients, involve them in the healing process and emphasize the importance of doctor-patient relationship; Treat the whole personnaturopathic medicine takes into account all aspects of an individual's health including physical, mental, emotional, genetic, environmental, social and spiritual 
factors; Prevention-naturopathic medicine emphasizes optimal wellness and the prevention of disease.

Treatment addresses the patient's underlying condition rather than the individual presenting symptoms. The naturopathic physician (ND) strives to thoroughly understand each patient's condition and view symptoms as the body's means of communicating and underlying imbalance. The main modalities the ND's utilize are diet, clinical nutrition, behavioral change, hydrotherapy, homeopathy, botanical medicine, physical medicine, pharmaceuticals and minor surgery ${ }^{[33,34]}$. Naturopathy can also be traced back to the European "nature cure," practiced in $19^{\text {th }}$ century, which was a system for treating disease with natural modalities such as water, fresh air, diet and herbs. In $20^{\text {th }}$-century developed in the U.S and Canada, combining nature cure, homeopathy, spinal manipulation and other therapies ${ }^{[35]}$. Naturopathy is most accurately viewed as a "whole system" medical practice ${ }^{[36,37]}$, a paradigm guiding the selection and prescription of complex, indivualized, multimodality treatments regimens. Naturopathy has defined reduction to single modality. Naturopaths will take care of reviewing dietary and lifestyle patterns and may offer prescriptions in these areas at a level of detail that exceeds what is commonly provided by allopathic primary care physicians. Naturopathic physicians having a good training and expertise in herbal medicine, which allopathic physicians generally lack. According to Ayurveda, the indigenous system of Indian medicine, which predates naturopathy by thousands of years, dairy products properly prepared, are a "stavic" food, of the highest order of nutritional value. Treatment modalities those utilized by the ND's include the diet and clinical nutrition, behavioral change, hydrotherapy, homeopathy, botanical medicine and physical medicine, also perform minor office procedures and surgery, administer vaccinations and prescribe many prescriptive drugs ${ }^{[38]}$. Proper nutrition is the foundation of a naturopathic practice; here the food is utilized for both health promotion and disease prevention. The ND's recommended diets to each patient a set of balanced whole-foods diet rich in fruits, vegetable, whole grains, legumes, wild caught fish, lean animal proteins, whole dairy products. Although acupuncture and chiropractic care have achieved a set of measure of acceptance within mainstream medicine, the integrative role of naturopathy has yet to be well specified.

\section{Yoga:}

Yoga is specified as a mind and body therapy comprising of various breathing and relaxation techniques. This includes the meditation, postures and phrase recitation to improve the mental illness and physical illness ${ }^{[39]}$. Yoga nidra is a type of relaxation technique, a form of sleep to attain a form of blissful awareness which is not attained with other forms of yoga ${ }^{[40]}$. Iyengar yoga usually helps in postural alignment and movement; this usually promotes the use of blocks, mats, blankets and belts $^{[41]}$. Hatha yoga incorporates yoga postures (asanas), breathing exercises (pranayama's), meditation (dyana), balancing of opposite properties (heat/activation with cool/calming) for mental health development ${ }^{[42]}$, kripalu $^{[43]}$ and mindfulness based stress reduction ${ }^{[44]}$ also include in the Yoga. Sudarshankriya yoga helps in rhythmic breathing techniques performed within sitting posture eyes and mouth kept closed ${ }^{[45]}$. Yoga is used to treat various diseases and disorders like anxiety, depression, menustral irregularity, fibromyalgia, hypertension and stress also used in the treatment of cancer.

\section{Yoga in treatment of anxiety:}

Anxiety is most common psychiatric disorders with various comorbidities like depression, restless leg syndrome, cancer and hypertension. The higher costs of pharmacological therapies, gave rise to the use of alternative methods of treatment ${ }^{[46]}$. Yoga is used as a therapy to lower the heart rate and blood pressure in case of people suffering with anxiety ${ }^{[4]}$. Symptoms associated with anxiety include short and choppy breaths yoga is able to treat the warning signs of anxiety ${ }^{[47]}$. Yoga works because of the activation parasympathetic nervous system and increases the neurotransmitter Gamma aminobutyric acid (GABA) in the brain and thereby relieving anxiety. Yoga also decreases anxiety in patients with psychiatric disorders (anxiety, stress or depression). Yoga also helps to reduce the physiological and psychological responses to stress.

\section{Yoga in cancer therapy:}

The use of yoga as Complementary and alternative medicine (CAM) is proven to be beneficial. Most of the studies reported that yoga improved the physical and physiological symptoms, quality of life and markers of immunity of the patients providing a strong support for yoga's integration into conventional cancer care. Cancer affects the quality of life from its starting stage of 
diagnosis, progression, adverse effects of its treatment and diagnostic procedures by affecting the physical, physiological and emotional problems of patients ${ }^{[48-56]}$. Yoga interventions are beneficial in improving the adverse symptoms in cancer patients caused either by the disease or its treatment.

The greatest challenges of cancer care providers are: How to prevent the onset of cancer (transformation of a cell into a cancer cell); How to arrest its progression; How to cure cancer; How to maintain the survivors Quality of life (QOL).

The studies proved the evidence of the beneficial effects of yoga on the physical and the emotional health and QOL of cancer patients. Yoga prevents the tumorigenesis and progression and ultimately cures cancer. Cancer caregivers are trained and certified as the 'Yoga therapists' who have knowledge of the particular disease and understand the needs of the patients. Greater efforts must be made to integrate yoga as a main stream therapeutic program.

\section{Herbal medicine:}

Herbal medicines are the use of medicinal plants for the prevention and treatment of various diseases. Herbal medicine is mainly based on the remedies those containing active principles at very low concentrations, or relying on magical-energetic principles. Traditional medicines are sum total of practices based on theories, belief and experience of different cultures and times used in the maintenance of health mainly in the prevention, diagnosis, improvement and treatment of illness. The mind body medicine is considered as alternative or the complementary mode of traditional western medicine and various modes used are a part of the mind body connection. A successful treatment using this herbal medicines and the assessment of efficacy is not different from that of conventional medicine. Herbal medicinal plants are used in the treatment of various types of diseases such as Garlic (Allium sativum L.) used for the treatment of Influenza and Diarrhea etc.

\section{Herbal medicines in treatment of Diabetes Mellitus:}

Diabetes mellitus emerges due to various factorsinhibition of glucose absorption, up regulation of glucose transporters, increase in glucose uptake, glycogen metabolism, decreased insulin production, activation of the nuclear receptors peroxisome proliferator-activated receptors (PPARs), Also because of effects of some opioids on glucose homeostasis and antioxidants. Diabetes mellitus usually affects a set of 150 million people worldwide. Herbal medicines and their preparations are useful for the treatment of diabetes mellitus, 200 traditional plants and their bioactive constituents possess anti-diabetic property. Phytochemicals of various plants are said to be much effective as alternative medicines. Extracts of various medicinal plants act at different levels by inhibiting the glucose absorption from intestine, increasing the insulin secretion from pancreas, increasing the uptake of glucose by adipose and muscle cells, inhibiting the glucose production from hepatocytes ${ }^{[57]}$. Anti-diabetic effect of oral borapetol B compound isolates from the plant Tinospora crispa, helps by stimulating the insulin release. Stevioside obtained for the plant Stevia rebaudiana Bertoni increases the insulin sensitivity in 3T3-L1 adipocytes, also exerts the anti-hyperglycemic effect. Hence, herbal medicine as an alternative medicine helps in treating diabetes mellitus by various mechanisms of action.

\section{Massage therapy:}

Massage therapy is mainly used to manage a health condition or top increase the wellness, by manipulating the soft tissues of the body. Massage therapy is defined as the soft tissue manipulation mainly for the therapeutic purposes done by the trained therapists ${ }^{[58]}$. This therapy is mainly used to relieve pain. Massage therapy has been followed using the mechanical devices in addition to the therapists, applied to single or multiple body parts. Swedish/classical massage is the main type of massage therapy in several countries, clinical massage and sports massage is mainly used for muscle spasms. Other various massage therapies are Shiastu, Rolfing, reflexology and craniosacral therapy. Massage therapies are mainly used to treat several types of pain such as low back pain, shoulder pain, neck pain and pain from osteoarthritis of the knee and headache. Massage therapy is also known to be the adjunctive therapy to help prepare the patient for exercise or any other interventions. Massage therapy is rarely administered as main treatment.

\section{Massage therapy in cancer:}

Massage therapy has been used to relieve pain and other symptoms in cancer patients but the quality of evidence was very low. This therapy was used for the care of breast cancer patients by helping in the reduction of stress, anxiety, fatigue, depression and QOL. In patients suffering with lung cancer massage therapy is used as a care for those patients whose anxiety, pain is not 
controlled by usual care. Massage therapies should be of less pressure while being used in case of cancer patients.

\section{Massage therapy in baby growth:}

Massage therapy effects baby growth by weight gain in infants, 34 studies of massage therapy for the premature infants in the y 2017 has evaluated the effect of massage therapy on infants with improvement.

\section{Massage therapy in HIV/AIDS:}

Massage therapy shown a better response for quality of life, anxiety and depression in case of patients suffering with human immunodeficiency virus (HIV)/ Acquired immunodeficiency syndrome (AIDS). In the y 2010 the review of 4 studies has proven that quality of life for people has been increased in case of HIV/ AIDS patients, in the y 2013 massage therapy has been proven for its use in reducing anxiety and depression in patients suffering with HIV/AIDS.

\section{Massage therapy in treatment of pain:}

Massage therapy was considered superior to relaxation, acupuncture and this therapy was considered to be equal to exercises and corsets. The acupressure/pressure point massage techniques provided more pain relief. Massage therapy is mainly used in case of chronic pain (a continuous recurrent pain that persists for longer than the normal time of healing $)^{[59]}$. There was an evidence for the craniosacral massage in managing tension type Headache pain, sub-acute and chronic nonspecific low back pain management, a preliminary support for pain relief in carpal tunnel syndrome and equivocal support for the analgesic effects in fibromyalgia was reported with massage therapy.

\section{Alternative medicine therapies on Muscular Atrophy:}

Muscle atrophy is defined as the reduction in the muscle mass that includes the partial or complete wasting away from the muscle. The functional and morphological changes those caused by the muscular atrophy usually result in decreased protein content, muscle fiber cross sectional area, muscle strength and increased insulin resistance ${ }^{[60]}$. The muscle atrophy also occurs in cachexia, a comorbidity of abnormal conditions such as cancer, AIDS, congestive heart failure and chronic obstructive pulmonary disease, also occurs in sarcopenia, a decreases in muscle mass and strength associated with aging ${ }^{[61]}$. The symptoms of muscular atrophy are treated using acupuncture, herbal medicine and chuna treatment. Various CAM therapies for muscular atrophy have also been reported. The CAM therapy promoted protein synthesis and inhibited the degradation by enhancing the phosphoinositide 3-kinase (PI3K)/protein kinase B (Akt)/mammalian target of rapamycin (mTOR) signaling pathway. CAM alleviated skeletal muscle metabolism through modulating mitochondrial biogenesis factors, thereby prevention of muscle loss, it also restored the expression of proteins involved in the cell apoptotic pathway and autophagy pathway in the various models of muscular atrophy.

\section{Alternative medicine in management of osteoarthritis:}

Osteoarthritis is a degenerative and progressive disease mainly affecting the joint cartilage and the subchondrial bone which increases with age ${ }^{[62]}$. A large number of different therapies have been described in the medical literature in relation to the treatment of osteoarthritis. The non-pharmacological modalities include education about the objectives of treatment and changes in life style, such as exercise and weight reduction ${ }^{[63]}$. There was proven evidence that capsaicin gel and S-adenosyl methionine were effective in the management of osteoarthritis ${ }^{[64]}$, Indian Frankincense, methyl sulphonyl methane and rose hip may also be effective. Homeopathic remedies in the treatment of osteoarthritis were tested. Local application of a homeopathic remedy (Spiroflor), which contains Symphytum officinale R., Toxicondendron and Ledum palutres or piroxicam gel $(0.5 \%)$. Only a set of minor adverse symptoms were reported among persons taking the homeopathic remedies. Alternative medicines are also useful in the treatment of Rheumatoid arthritis, osteoarthritis and fibromyalgia.

\section{BENEFITS OF ALTERNATIVE MEDICINE}

The most frequently endorsed benefits are get relief for my symptoms, the pain or discomfort is less or goes away, feel better and the treatment works better for my particular health problem than standard medicines. The treatment promotes health rather than just focusing on illness. Narendra Modi prescribes Ayush traditional ways and endorsed traditional ways to boost immune system, also suggested people to follow the protocol of ministry of Ayurveda, Yoga and Naturopathy, Unani, Siddha and Homeopathy to fight with various diseases. Narendra Modi said that Ayurveda is not just an alternative today but is one of the key bases 
of the Country's health policy. Bhadra Ayurveda and panchakarma are the permanent cure for joint pains and determined to be expert treatment for Arthritis, Spondylitis and all types of joint pains. Oscar Fernandes a Congress leader shares story of cow urine curing cancer, known as the virtues of "gaumutra". The virtues of Indian systems of medicine were also praised and said that knee joint pain has been cured completely with the help of "vajrasana" in yoga stating that Yoga is our wealth. Our Indian systems of medicine will provide a lot of reliefs even before going to a doctor. Cow cuddling is believed to promote positivity and reduce stress by boosting oxytocin in humans, the calming effects of curling with a pet or emotional support animal, it seems, are accentuated when cuddling with larger mammals. Embracing Cows, or "KoeKnuffelen" in Dutch, is more than a cute wellness trend. With immense mental health benefits, the practice has growing global appeal. Tai chi, acupuncture and osteopathic manipulation therapy are all the viable options for improving mobility and reducing the pain symptomatology. CAM has a large and growing in this country and all over the world. The increasing use of CAM represents significant shift in the health care received by the populations of industrialized countries.

\section{CONCLUSION}

Alternative medicine has become popular and many of the professionals working in occupational and environmental medicine feel the need to learn about it and implement it. Many adults and children, several patients, ambulatory patients use alternative medicine in addition to the traditional therapies. A wide spread of increase in the use of alternative medicines and practicing has been noted in the recent years. There is a need for the alternative medicine industry and also its health professionals to improve its self regulation and standards. The public has gained accurate information about the alternative medicines within the internet and alternative medicine websites where the safety and efficacy information is clearly presented. Several challenges have been set for the researchers to find more appropriate ways of studying the effectiveness of complementary and alternative therapies .Various alternative therapies are assigned to treat various diseases, most of the alternative therapies are used to treat various diseases which could be mentioned as problem with the conventional medicine therapy. CAM is and increasingly important system which implies good patient compliance. Hence alternative medicine serves as the important therapies in treatment of several diseases and increases the level of acceptance in patients which is reported to be a problem with some conventional therapies and serves to a greater degree within some or all of its principles.

\section{Conflict of interests:}

The authors declared no conflicts of interest.

\section{REFERENCES}

1. Berkenwald A. In the name of medicine. Ann Intern Med 1995;128:246-50.

2. Eisenberg DM, Kessler RC, Foster C, Horlock FE, Calkins DR, Delbanco TL. Unconventional medicine in the United States. prevalence, costs and patterns of use. N Engl J Med 1993;328(4):246-52.

3. Goldbeck-Wood S, Dorozynski A, Lie Lg,. Complementary medicine is booming worldwide. BMJ 1996;313(7050):131-3.

4. MacLennan AH, Wilson DH, Taylor AW. Prevalence and cost of alternative medicine in Australia. Lancet 1996;347(9001):56973.

5. CTV/Angus Reid Group poll. Use of alternative medicines and practicies. Angus Reid Group, Inc 1997.

6. Saul G. Alternative Medicine and internet. Paediatr Child Health 1999;4(8):539-41.

7. Laxminkantham V. Origin of Human Past. Bharathiya Vidya Bhavan, Bombay 1999.

8. Kutumbaih P. Ancient Indian Medicine. Orient Longman's Bombay 1962.

9. Firth WJ. Chaos-predicting the unpredictable. BMJ 1991;303(6817):1565-68.

10. Dharmapal. Indian Science and Technology in the 18th century, 1983, Academy of Gandhian Studies, Hyderabad.

11. Hegde BM: Angina an Indian Disease. J Assoc Physicians India 1999;47:440-2.

12. Frawley David. Ayurveda and the Mind. 2000. Motial Banarsidas. Ayurveda the mother of all wisdoms.

13. Swayne J. International Dictionary of Homeopathy 2002;54:577-82.

14. Ernst E. Homeopathy, past present future. Br J Clin Pharmacol 1997;44(5):435-7.

15. Linde K, Clausius N, Ramirez G, Melchart D, Eitel F, Hedges $\mathrm{LV}$, et al. Are the clinical effects of homoeopathy placebo effects? A meta-analysis of placebo-controlled trials. Lancet 1997;350:834-43.

16. Bornhoft JM, Strabala KW, Wortman TD, Lehman AC, Oleynikov D, Farritor SM. Stereoscopic visualization and haptic technology used to create a virtual environment for remote surgery-biomed 2011. Biomed Sci Instrum 2011;47:7681.

17. Reilly DT, Taylor MA, McSharry C, Aitchison T. Is homoeopathy a placebo response? Controlled trial of homoeopathic potency, with pollen in hayfever as model. Lancet 1986;2(8512):881-6.

18. Witt CM, Ludtke R, Baur R, Willich SN. Homeopathic medical practice: long-term results of a cohort study with 3981 patients. BMC Public Health 2005;5(1):1-8.

19. Frass M, Dielacher C, Linkesch M, Endler C, Muchitsch I, Schuster E, et al. Influence of potassium dichromate on tracheal secretions in critically ill patients. Chest 2005;127(3):936-41.

20. Nimgulkar CC, Patil SD, Kumar BD. Anti-asthmatic and antianaphylactic activities of Blatta orientalis mother tincture. Homeopathy 2011;100(3):138-43.

21. Enbergs H. Effects of the homeopathic preparation Engystol 
on interferon-gamma production by human T-lymphocytes. Immunol Invest 2006;35(1):19-27.

22. Rosted P. Introduction to acupuncture in dentistry. Br Dent $\mathbf{J}$ 2000;189(3):136-40.

23. Ernst E, Pittler MH, Stevinson C, White A. The desktop guide to complementary and alternative medicine: an evidence-based approach. Mosby International Ltd 2001;98(3):330-7.

24. Stux G, Pomeranz G. Scientific Basis of Acupuncture: Acupuncture Textbook and Atlas. Heidelberg: Springer Verlag; 1987medical acupuncture 2014;26(6).

25. Sierpina VS, Frenkel MA. Acupuncture: a clinical review. South Med J 2005;98(3):330-7.

26. NIH Consensus Statement: Acupuncture. South Med J 2005;98(3):330-7.

27. Helms J. Acupuncture Energetics. Berkeley, CA, Medical Acupuncture Publishers, Acupuncture: A Clinical Review 1996;(98);3300-7.

28. Cho ZH, Chung SC, Jones JP, Park JB, Park HJ, Lee HJ, et al. New findings of the correlation between acupoints and corresponding brain cortices using functional MRI. Proc Natl Acad Sci 1998;95(5):2670-3.

29. Traditional Chinese Medicine/Acupuncture. Southern Medical Association 2005;98(3).

30. World Health Organization list of common conditions treatable by Chinese Medicine and Acupuncture. Available at: http:// tcm.health-info.org/WHO-treatment-list.htm; 2003.

31. Helms J. An overview of medical acupuncture. Altern Ther Health Med 1998;4:35-45.

32. House of Delegates position paper: definition of naturopathic medicine [monograph on the Internet]. Washington, DC: American Association of Naturopathic Physicians; amended 2011.

33. Sara A. Fleming, Nancy C. Gutknecht, Bastyr University 2010;37(1):119-36.

34. The American Association of Naturopathic Physicians. PMC 2010:1-16.

35. Kirchfeld F, Boyle W. Nature Doctors: Pioneers in Naturopathic Medicine. Med Biol 1994;37(1):119-36.

36. Ritenbaugh C, Verhoef M, Fleishman S, Boon H, Leis A. Whole systems research: a discipline for studying complementary and alternative medicine. Altern Ther Health Med 2003;9:32-6.

37. Elder C, Aickin M, Bell IR, Fønnebø V, Lewith GT, Ritenbaugh $\mathrm{C}$, et al. Methodological challenges in whole systems research. J Altern Complement Med 2006;12(9):843-50.

38. Fleming and Gutknecht.Washington Association of Naturopathic Physicians;2009. Available at: http://www.wanp. org $/ \mathrm{mc} /$ page.do?sitePageId=58070\&orgId=wanp.

39. Rocha KK, Ribeiro AM, Rocha KC, Sousa MB, Albuquerque $\mathrm{FS}$, Ribeiro $\mathrm{S}$, et al. Improvement in physiological and psychological parameters after 6 months of yoga practice. Conscious Cogn 2012;21(2):843-50.

40. Rani K, Tiwari S, Singh U, Agrawal G, Ghildiyal A, Srivastava N. Impact of yoga nidra on psychological general wellbeing inpatients with menstrual irregularities: a randomized controlled trial. Int J Yoga 2011;4:20-25.

41. Harner H, Hanlon AL, Garfinkel M. Effect of Iyengar yoga on mental health of incarcerated women: a feasibility study. Nurs Res 2010;59(6):389-99.

42. Gururaja D, Harano K, Toyotake I, Kobayashi H. Effect of yoga on mental health: Comparative study between young and senior subjects in Japan. Int J Yoga 2011;4(1):7.

43. Carson JW, Carson KM, Jones KD, Bennett RM, Wright CL, Mist SD. A pilot randomized controlled trial of the Yoga of Awareness program in the management of fibromyalgia. Pain 2010;151(2):530-9.

44. Schmidt S, Grossman P, Schwarzer B, Jena S, NaumannJ, Walach H. Treating fibromyalgia with mindfulness- based stressreduction: results from a 3-armed randomized controlled trial. Pain. 2011;152(2):361-369.

45. Telles S, Singh N, Joshi M, Balkrishna A. Post-traumatic stress symptoms and heart rate variability in Bihar flood survivors following yoga: a randomized controlled study. BMC Psychiatry 2010;10(1):1-0.

46. Katzman MA, Vermani M, Gerbarg PL, Brown RP, Iorio $\mathrm{C}$, Davis $\mathrm{M}$, et al. A multicomponent yoga-based, breath intervention program as an adjunctive treatment in patients suffering from generalized anxiety disorder with or without comorbidities. Int J Yoga 2012;5(1):57-65.

47. Thygeson MV, Hooke MC, Clapsaddle J, Robbins A, Moquist K. Peaceful play yoga: serenity and balance for children with cancer and their parents. J Pediatr Oncol Nurs 2010;27(5):27684.

48. Allard P, Maunsell E, Labbé J, Dorval M. Educational interventions to improve cancer pain control: A systematic review. J Palliat Med 2001;4:191-203.

49. Brændengen $\mathrm{M}$, Tveit $\mathrm{KM}$, Bruheim $\mathrm{K}$, Cvancarova $\mathrm{M}$, Berglund A, Glimelius B. Late patient-reported toxicity after preoperative radiotherapy or chemoradiotherapy in nonresectable rectal cancer: Results from a Randomized phase III study. Int J Radiat Oncol Biol Phys 2011;81(4):1017-24.

50. Bruheim K, Guren MG, Skovlund E, Hjermstad MJ, Dahl $\mathrm{O}$, Frykholm G, et al. Late side effects and quality of life after radiotherapy for rectal cancer. Int $\mathrm{J}$ Radiat Oncol Biol Phys 2010;76(4):1005-11.

51. McGuire DB. Occurrence of cancer pain. J Natl Cancer Inst Monogr 2004;2004(32):51-6.

52. Ram PAgarwal, Adi Marko-Afek. NIH NCI. Side Effects; 2015.

53. Oh D, Huh SJ, Nam H, Park W, Han Y, Lim DH, et al. Pelvic insufficiency fracture after pelvic radiotherapy for cervical cancer: Analysis of risk factors. Int $\mathrm{J}$ Radiat Oncol Biol Phys 2008;70(4):1183-8.

54. Quasthoff S, Hartung HP. Chemotherapy-induced peripheral neuropathy. J Neurol 2002;249(1):9-17.

55. Gorin SS, Krebs P, Badr H, Janke EA, Jim HS, Spring B, et al. Meta-analysis of psychosocial interventions to reduce pain in patients with cancer. J Clin Oncol 2012;30(5):539-47.

56. Van den Beuken-van Everdingen MH, De Rijke JM, Kessels AG, Schouten HC, Van Kleef M, Patijn J. Prevalence of pain in patients with cancer: a systematic review of the past 40 years. Ann Oncol 2007;18(9):1437-49.

57. El-Abhar HS, Schaalan MF. Phytotherapy in diabetes: Review on potential mechanistic perspectives. World journal of diabetes 2014;5(2): 176 .

58. Field TM. Massage therapy effects. Am Psychol 1998;53(12):1270-81.

59. Merskey HE. Classification of chronic pain: Descriptions of chronic pain syndromes and definitions of pain terms. Pain 1986.

60. Frontera WR, Ochala J. Skeletal muscle: a brief review of structure and function. Calcif Tissue Int 2015;96(3):183-95.

61. Cohen S, Nathan JA, Goldberg AL. Muscle wasting in disease: molecular mechanisms and promising therapies. Nat Rev Drug Discov 2015;14(1):58-74.

62. March LM, Bagga H. Epidemiology of osteoarthritis in Australia. Med J Aust 2004;180(5):S6.

63. Woolf AD, Pfleger B. Burden of major musculoskeletal conditions. Bull World Health Organ 2003;81:646-56.

64. Zhang W, Moskowitz RW, Nuki G, Abramson S, Altman RD, Arden $\mathrm{N}$, et al. OARSI recommendations for the management of hip and knee osteoarthritis, Part II: OARSI evidencebased, expert consensus guidelines. Osteoarthritis Cartilage 2008;16(2):137-62. 